\title{
Factores externos y elección de la carrera profesional en estudiantes de Derecho, Arequipa 2011
}

External factors and the Professional Career selection in Law students Arequipa 2011.

\author{
Sandro Peralta Arotaype, Darwin Moscoso Montoya \\ Universidad Católica de Santa María. Arequipa Perú.
}

\section{INFORMACIÓN}

\section{Historia del Artículo}

Recepción: $15 / 11 / 2018$

Revisión: 02/02/2019

Aceptación: 15/03/2019

\section{Palabras Clave}

Factores externos, elección de la carrera.

\section{Key Words}

External factors, election of the career choice

DOI

https://doi.org/10.35286/veritas. v20i1.226

\begin{abstract}
RESUMEN
El presente trabajo de investigación "Factores externos y elección de la carrera profesional en estudiantes de Derecho, Arequipa 2011". Objetivo: Determinar qué factores externos son los que intervienen en la elección de la carrera profesional, al observar que, en la localidad, una de las carreras con mayor demanda es la de Derecho. Las variables a investigar fueron: "Factores externos de la carrera profesional (variable 1) y la elección de la carrera profesional (variable 2). Hipótesis: Se partió de la hipótesis que el entorno socio cultural ético-moral científico son los factores externos que más consideran los estudiantes del primer año del Programa Profesional de Derecho. La técnica empleada fue la encuesta colectiva y estructurada, y el instrumento, un cuestionario tipo Escala de Likert de 55 afirmaciones, con alternativas de respuesta para marcar. Conclusiones: Los factores externos de vínculo familiar $(52,2 \%)$ y sistema educacional $(58 \%)$ son los que tienen el nivel más ALTO de presencia dentro de la carrera profesional de Derecho que eligieron los alumnos encuestados.
\end{abstract}

\begin{abstract}
The present research "External factors and the professional career selection in law students Arequipa 2011". Objetive: Determine what external factors are the ones that in the professional career selection, taking into account that nowaday one of the most demanding professional career in law. The variables the research were: External factors of the professional career (variable 1) an the election of the professional career (variable 2). Hypothesis: we started with the Hypothesis that the social cultural, ethical moral and the scientific environment are considered factors by the students of the first professional program. The applied technique was a colective and structured survey and the instrument was a questionary likert scale of 55 affirmations with alternatives of to choose. Conclusions: the external factors of familiar link were $(52,2 \%)$, and the educational system $(58 \%)$ they are the ones that have the highest level presence into the professional career of law that were elected by the students who did the survey.
\end{abstract}

\section{INTRODUCCIÓN}

En el proceso educativo a nivel superior, la formación inicial del alumno es importante para así garantizar una mejora en la calidad del aprendizaje, y es también pieza clave para el desarrollo personal de éste. Aceptarlo dentro de una carrera profesional no es únicamente fijar criterios administrativos, pedagógicos, sino establecer criterios que ayuden a reflexionar sobre la propia práctica y proponer acciones basadas en una orientación e información que les orienten para llevar una buena formación en el desarrollo profesional posterior de la carrera elegida, que permita que el nuevo estudiante sea lo bastante responsable para poder afrontar los retos del quehacer educativo. Por todo lo mencionado, este trabajo de investigación tiene como objetivo contribuir a que se tenga una mejor información que conlleve a replantear el proceso de elección de la carrera profesional.

Al terminar los estudios de nivel secundaria, se abre la universidad con sus múltiples carreras: la militar, el campo, la industria, el comercio, un empleo, una carrera técnica,

Correspondencia:

Sandro Peralta Arotaype

santrex@hotmail.com el arte, etc. Se abren ante sí perspectivas más amplias que las carreras mismas: las formas de vida (política, espiritual, social, etc.) Todo esto se presentará al iniciar su vida en forma más personal e independiente.

Muchos jóvenes, por desgracia, no toman en serio esta situación, desperdician el tiempo que tuvieron para obtener información sobre las distintas carreras que existen, los programas, las escuelas y no aprovechan un espacio de tiempo para autoanalizarse y reflexionar sobre cuál carrera sería la mejor para ellos. Es entonces cuando la elección de carrera se convierte en un conflicto, ya que cuando apenas van saliendo de su educación obligatoria secundaria, toman conciencia sobre lo que quieren estudiar, y se hallan presionados por el tiempo o porque todavía no saben qué carrera elegir.

\section{METODOLOGÍA}

\section{Técnica}

La técnica empleda fue la encuesta aplicada a cada unidad de estudio en forma indicvidual.

\section{Instrumento}

El instrumento aplicado fue un cuestionario elaborado por los investigadores, que era de tipo Escala de Likert de 55 afirmaciones, con opciones de respuestas (totalmente de acuerdo, de acuerdo, indeciso, en desacuerdo, totalmente 
en desacuerdo) con puntajes respectivos de $(5,4,3,2,1)$ que el estudiante tuvo que elegir. Midió los indicadores de la variable 1: Vínculo familiar, Sistema educacional, Entorno socio cultural ético-moral científico, Contexto socio económico expectativas laborales y Presencia de los medios de comunicación social.

De la variable 2, los indicadores fueron: Identificar y analizar la situación, Comparar las carreras, Determinar la elección, Evaluar riesgos, Seleccionar la mejor alternativa e Instrumentar la decisión.

\section{Población y muestra}

El Universo estuvo representado por los estudiantes del primer año del Programa Profesional de Derecho de la Universidad Católica de Santa María de Arequipa, que fueron en total 138 entre hombres y mujeres.

Se realizaron descripciones de la situación del problema del estudio y la comprobación de la hipótesis en base a los resultados de las pruebas estadísticas empleadas.

\section{RESULTADOS}

Tabla 01: Factores externos de la Carrera Profesional

\begin{tabular}{ccc}
\hline Vínculo familiar & fi & $\%$ \\
\hline Alto & 72 & 52,2 \\
Medio & 36 & 26,1 \\
Bajo & 30 & 21,7 \\
Total & 138 & 100,0 \\
\hline
\end{tabular}

Tabla 02: Entorno socio cultural, ético y científico

\begin{tabular}{ccc}
\hline $\begin{array}{c}\text { Entorno socio cultural, } \\
\text { ético y científico }\end{array}$ & $\mathrm{fi}$ & $\%$ \\
\hline Alto & 40 & 29,0 \\
Bajo & 8 & 5,8 \\
Medio & 90 & 65,2 \\
Total & 138 & 100,0 \\
\hline
\end{tabular}

\section{Elección de la carrera profesional}

Tabla 03: Comparar las carreras

\begin{tabular}{ccc}
\hline Comparar las carreras & $\mathrm{fi}$ & $\%$ \\
\hline Alto & 13 & 9.4 \\
Bajo & 31 & 22.5 \\
Medio & 94 & 68.1 \\
Total & 138 & 100.0 \\
\hline
\end{tabular}

\section{DISCUSIÓN}

El presente trabajo de investigación que lleva por título: "Factores externos y elección de la carrera profesional en los estudiantes del primer año del Programa Profesional de Derecho, Universidad Católica de Santa María, Arequipa 2011, ha permitido saber cómo han realizado su elección de la carrera profesional estos estudiantes.

Dentro de los factores externos de la carrera profesional, se puede ver que el entorno familiar es importante dentro de la actividad educativa de los jóvenes, ya que el $52,2 \%$ de ellos dice que tiene una alta influencia en las decisiones que toma. Resultado parecido al de Martínez (1993) encontrado en México en donde la familia es la primera expresión de la sociedad que influye y determina gran parte de la conducta de los adolescentes. Este periodo de la vida, como todo fenómeno humano, tiene su exteriorización característica dentro del marco familiar donde se desarrolla.

El entorno sociocultural, ético y científico son medianamente influyentes en las diversas decisiones que toma el adolescente al acabar su vida estudiantil en el colegio y decidirse optar por una carrera profesional a estudiar. "Tambien encontramos que el factor económico impide a nuestros estudiantes elegir libremente su carrea $\mathrm{y}$, generalmente se conforman con realizar estudios para los cuales no tienen interés, ni aptitudes, ni cuentan con las características personales que permitan su desarrollo como estudiantes y después como profesionistas.” (Martínez, 1993).

Los alumnos consideran que se tuvo que determinar la elección de escoger la carrera de Derecho luego de un proceso de pensar, meditar y buscar información de varias posibles alternativas de carreras profesionales en donde realizar estudios en el nivel superior, "Es importante analizar si la carrera congenia con los intereses de cada uno, gustos, habilidades y si se está dispuesto a invertir tiempo y esfuerzo en ella. Asimismo, dialogar con los padres, profesores y amigos sobre las inquietudes y escuchar las perspectivas que otros tienen sobre el tema."

\section{REFERENCIAS BIBLIOGRÁFICAS}

1. Canfux, V. y Rojas, A. (2003). Una metodología para el estudio de las condiciones que influyen en la dedicación al estudio de los estudiantes. Revista Cubana de Educación Superior. No 13, pp. 50-54.

2. Colque, V. (2003). Metodología de la investigación. Arequipa: Universidad Católica Santa María.

3. Corrales, J. (2003. Psicopedagogía contemporánea. Arequipa: Imprenta Gráfica Integral.

4. Dubois, J. (2005). Diccionario de Términos. Madrid: Alhambra

5. Ferrater, J. (2004). Diccionario de Filosofía. Barcelona: Montecasino

6. Frente estudiantil de psicología y RR.II. y PP.(FEPSI-R). (1999). Bases neuroanatómicas y neurofisiológicas del psiquismo humano (esbozo para la construcción de una psicología científica). Escuela Profesional de Psicología. Arequipa: Universidad Nacional de San Agustín.

7. Hernández, R.; Fernández, C. y Baptista, P. (2014). Metodología de la investigación. Sexta edición. México: McGraw-Hill education.

8. Ortiz, P. (2006). El sistema de la personalidad. Lima: Centro Gráfico Comercial Orion S.R.L.

9. Petrovsky, A. (2006) Psicología general. Ciudad de La Habana: Editorial Pueblo y Educación.

10. Real Academia Española. (2005). Diccionario de la Lengua Española. 21 ${ }^{\mathrm{a}}$ ed. España: Espasa Calpe, S.A.

11. Zevallos, A. (2004). Defendiendo la salud mental. Lima. Asociación de Protección al Enfermo Mental Crónico. Recuperado de: http://andreszevallos. blogspot.com/2008/11/triptico.html 